\title{
THE LIMIT CYCLES OF DISCONTINUOUS PIECEWISE LINEAR DIFFERENTIAL SYSTEMS FORMED BY CENTERS AND SEPARATED BY IRREDUCIBLE CUBIC CURVES II
}

\author{
REBIHA BENTERKI ${ }^{1}$, LOUBNA DAMENE ${ }^{2}$ AND JAUME LLIBRE ${ }^{3}$
}

\begin{abstract}
In this paper we provide a lower bound for the maximum number of crossing limit cycles of some class of planar discontinuous piecewise linear differential systems formed by centers and separated by an irreducible algebraic cubic curve. First we prove that the systems constituted by three zones can exhibit $0,1,2,3$ or 4 crossing limit cycles having four intersection points with the cubic of separation. Second we prove that the systems constituted by two zones can exhibit 0,1 , or 2 crossing limit cycles having four intersection points with the cubic of separation.
\end{abstract}

\section{INTRODUCTION}

1.1. Classification of the irreducible cubic polynomials. A cubic curve is the set of points $(x, y) \in \mathbb{R}^{2}$ satisfying $P(x, y)=0$ for some polynomial $P(x, y)$ of degree three. This cubic is irreducible (respectively reducible) if the polynomial $P(x, y)$ is irreducible (respectively reducible) in the ring of all real polynomials in the variables $x$ and $y$.

A point $\left(x_{0}, y_{0}\right)$ of a cubic $P(x, y)=0$ is singular if $P_{x}\left(x_{0}, y_{0}\right)=0$ and $P_{y}\left(x_{0}, y_{0}\right)=$ 0 . A cubic curve is singular if it has some singular point, as usual here $P_{x}$ and $P_{y}$ denote the partial derivaties of $P$ with respect to the variables $x$ and $y$ respectively.

A flex of an algebraic curve $C$ is a point $p$ of $C$ such that $C$ is nonsingular at $p$ and the tangent at $p$ intersects $C$ at least three times. The next theorem characterizes all the irreducible cubic algebraic curves.

Theorem 1. The following statements classify all the irreducible cubic algebraic curves.

(a) A cubic curve is nonsingular and irreducible if and only if it can be transformed with affine transformations into one of the following two curves;

$$
\begin{array}{ll}
c_{1}(x, y)=y^{2}-x\left(x^{2}+b x+1\right)=0 & \text { with } b \in(-2,2), \text { or } \\
c_{2}(x, y)=y^{2}-x(x-1)(x-r)=0 & \text { with } r>1 .
\end{array}
$$

2010 Mathematics Subject Classification. Primary 34C29, 34C25, 47H11.

Key words and phrases. limit cycles, discontinuous piecewise linear differential systems, linear differential centers, irreducible cubic curves. 
(b) A cubic curve is singular and irreducible if and only if it can be transformed with affine transformations into one of the following three curves:

$$
\begin{array}{ll}
c_{3}(x, y)=y^{2}-x^{3}=0, & \text { or } \\
c_{4}(x, y)=y^{2}-x^{2}(x-1)=0, & \text { or } \\
c_{5}(x, y)=y^{2}-x^{2}(x+1)=0 . &
\end{array}
$$

Statement (a) of Theorem 1 is proved in Theorem 8.3 of the book [4] under the additional assumption that the cubic has a flex, but in section 12 of that book it is shown that every nonsingular irreducible cubic curve has a flex. While statement (b) of Theorem 1 follows directly from Theorem 8.4 of [4].

1.2. Crossing limit cycles. For $k=1, \ldots, 5$ let $C_{k}$ be the five classes of planar discontinuous piecewise linear differential systems formed by centers and separated by the irreducible cubic curve $c_{k}(x, y)=0$, or simply the irreducible cubic curve $c_{k}$.

Piecewise linear differential systems appear in the characterization of many real processes such as switches in electronic circuits, see for instance $[2,12,13]$.

In the qualitative theory of piecewise linear differential systems one of the important and difficult problems is the determination of the existance and the number of limit cycles. We recall that a crossing periodic orbit, is a periodic orbit of a piecewise linear differential system with a cubic separation curve $c_{k}$ with at least two points in $c_{k}$. If this periodic orbit is isolated we call it a crossig limit cycle. In recent years, much progress has been made in studying the existance and lower and upper bounds of crossing limit cycles of piecewise linear differential systems, see for example $[1,5,6,7,8,9,15,17,18,19]$.

There are previous results on the number of crossing limit cycles of discontinuous piecewise linear differential centers separated by a curve $\Sigma$. More precisely, if $\Sigma$ is one straight line then such systems have no crossing limit cycles see $[14,16]$. If $\Sigma$ is a conic the number of crossing limit cycles have been studied in [10], and if $\Sigma$ is a reducible cubic curve the number of crossing limit cycles have been analyzed in [11].

In [3] we started the study of crossing limit cycles of the discontinuous piecewise linear differential centers in $\mathbb{R}^{2}$ separated by an irreducible algebraic cubic curve. We proved that these differential systems only can exhibit at most three crossing limit cycles having two intersection points with the cubic of separation.

The objective of this paper is to provide lower bounds for the maximum number $\mathcal{N}$ of crossing limit cycles for the planar discontinuous piecewise linear differential centers which intersect the irreducible cubic curves $c_{i}$, with $i=1 \ldots 5$, and have four points of intersection with the cubic of separation. First, we study the number $\mathcal{N}$ when the crossing limit cycles intersect the curves $c_{2}$ and $c_{5}$, in four points in three different zones. Second, we give $\mathcal{N}$ when the crossing limit cycles intersect $c_{i}$ with $i=1 \ldots 5$, in four points in two different zones.

Figures 1 and 2 show the different regions separated by the cubic curves $c_{i}$, with $i=1 \ldots 5$. 

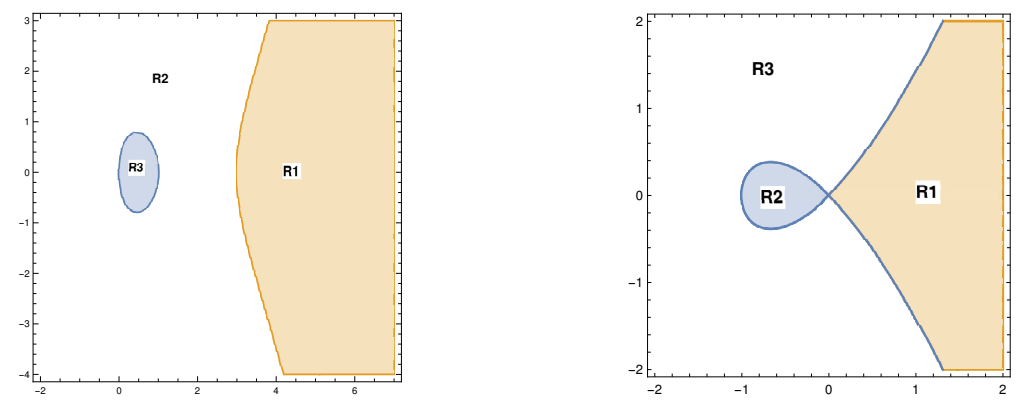

Figure 1. The three regions $R_{1}, R_{2}$ and $R_{3}$ of the plane separated by the curves $c_{2}$ on the left and $c_{5}$ on the right.
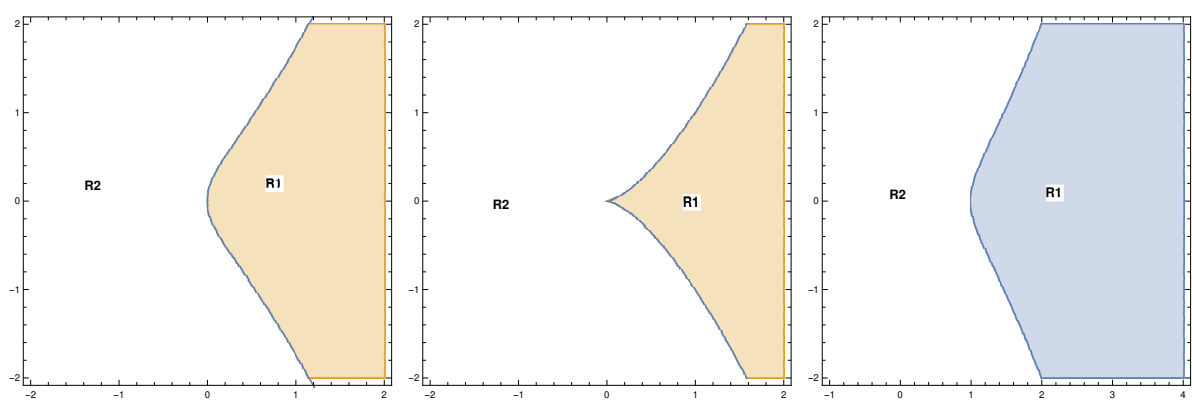

FiguRE 2. The two regions $R_{1}$ and $R_{2}$ of the plane separated by the curves $c_{1}$ on the left, $c_{3}$ on the middle and $c_{4}$ on the right.

1.3. Crossing limit cycles contained in three regions intersecting the two cubic curves $c_{2}$ and $c_{5}$ in four points. In this subsection we are interessted to provide lower bounds for the maximum number of crossing limit cycles of piecewise linear differential centers separated by the irreducible cubic curves $c_{2}$ or $c_{5}$, having four points of intersection with the cubic of separation. We note that such piecewise systems are formed by three pieces in each one there is a linear differential center.

Our first main result is the following.

Theorem 2. We study the crossing limit cycles contained in three regions of the discontinuous piecewise linear centers in the class $C_{2}$ or $C_{5}$ and having four points of intersection with the cubic of separation.

(a) There are systems in $C_{2}$ and in $C_{5}$ exhibiting exactly one crossing limit cycle which intersect $c_{2}$ or $c_{5}$ in four points. The class $C_{2}$ has one possible configuration see Figure 3 , while the class $C_{5}$ has two possible configurations see $\left(\mathcal{C}_{5}^{1}\right)$ and $\left(\mathcal{C}_{5}^{2}\right)$ of Figure 4.

(b) There are systems in $C_{2}$ and in $C_{5}$ exhibiting exactly two crossing limit cycles which intersect $c_{2}$ or $c_{5}$ in four points. The class $C_{2}$ has one possible configuration see Figure 5, while the class $C_{5}$ has two possible configurations see $\left(\mathcal{C}_{5}^{1}\right)$ and $\left(\mathcal{C}_{5}^{2}\right)$ of Figure 6. 
(c) There are systems in $C_{2}$ and in $C_{5}$ exhibiting exactly three crossing limit cycles which intersect $c_{2}$ or $c_{5}$ in four points. The class $C_{2}$ has one possible configuration see Figure 7 , while the class $C_{5}$ has two possible configurations see $\left(\mathcal{C}_{5}^{1}\right)$ and $\left(\mathcal{C}_{5}^{2}\right)$ of Figure 8.

(d) There are systems in $C_{2}$ and in $C_{5}$ exhibiting exactly four crossing limit cycles which intersect $c_{2}$ or $c_{5}$ in four points see $\left(\mathcal{C}_{2}\right)$ and $\left(\mathcal{C}_{5}\right)$ of Figure 9 for the classes $C_{2}$ and $C_{5}$, respectively.

Theorem 2 is proved in section 2 .

1.4. Crossing limit cycles contained in two regions intersecting the irreducible cubic curves $c_{i}$ in four points. Now we give our second main result which provides information on the number of crossing limit cycles of the discontinuous piecewise linear differential systems formed by two centers and intersect the cubic curves $c_{i}$, with $i=1 \ldots 5$ in four points.

Our second main result is the following.

Theorem 3. We study the crosssing limit cycles contained only in two regions of the discontinuous piecewise linear centers in the class $C_{k}$ for $k=1 \ldots 5$, and having four intersection points with the cubic of separation.

(a) There are systems in $C_{k}$ exibiting exactly one crossing limit cycle intersecting the cubic curves $c_{i}$ in four points. The classes $C_{1}, C_{3}$ and $C_{4}$ have one possible configuration, see $\left(\mathcal{C}_{1}\right),\left(\mathcal{C}_{3}\right)$ of Figure 10 and $\left(\mathcal{C}_{4}\right)$ of Figure 12 , respectively. The classes $C_{2}$ and $C_{5}$ have two possible different configurations see $\left(\mathcal{C}_{2}^{1}\right)$ and $\left(\mathcal{C}_{2}^{2}\right)$ of Figure 11 , and $\left(\mathcal{C}_{5}^{1}\right)$ and $\left(\mathcal{C}_{5}^{2}\right)$ of Figure 13 .

(b) There are systems in $C_{k}$ exibiting exactly two crossing limit cycles intersecting the cubic curves $c_{i}$ in four points. The classes $C_{1}, C_{3}$ and $C_{4}$ have one possible configuration see $\left(\mathcal{C}_{1}\right),\left(\mathcal{C}_{3}\right)$ and $\left(\mathcal{C}_{4}\right)$ of Figure 14 , respectively. The classes $C_{2}$ and $C_{5}$ have two possible different configurations see $\left(\mathcal{C}_{2}^{1}\right)$ and $\left(\mathcal{C}_{2}^{2}\right)$ of Figure 15 for the class $C_{2}$, and $\left(\mathcal{C}_{5}^{1}\right)$ and $\left(\mathcal{C}_{5}^{2}\right)$ of Figure 16 for the class $C_{5}$.

Theorem 3 is proved in section 3 .

\section{Proof of Theorem 2}

Proof of statement (a) of Theorem 2. First we prove the statement for the class $C_{2}$. We consider the first linear differential center in the region $R_{1}$

$$
\dot{x}=-\frac{x}{4}-\frac{5 y}{16}+\frac{5}{4}, \quad \dot{y}=x+\frac{y}{4}+\frac{1}{2},
$$

this system has the first integral

$$
H_{1}(x, y)=4\left(x+\frac{y}{4}\right)^{2}+8\left(\frac{x}{2}-\frac{5 y}{4}\right)+y^{2} .
$$

The second linear differential center in the region $R_{2}$ is

$$
\dot{x}=\frac{1}{56}(14 x-7 y-79), \quad \dot{y}=x-\frac{y}{4}-\frac{211}{64},
$$




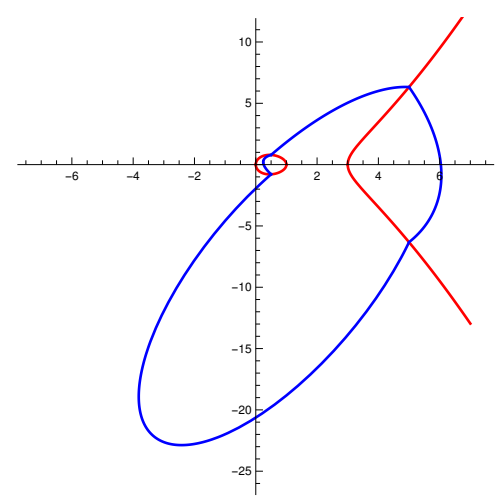

FiguRE 3. The unique limit cycle of the discontinuous piecewise linear differential system (1)-(3) contained in three zones.

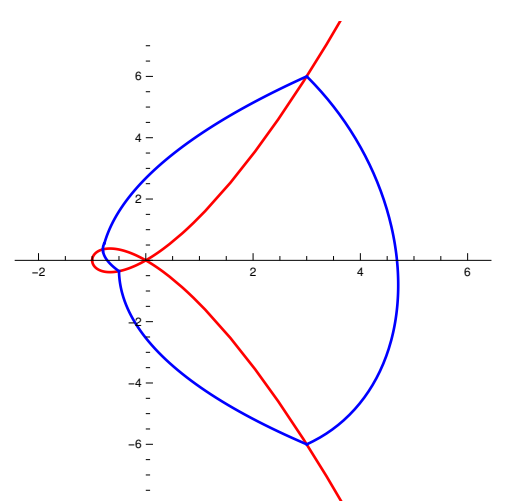

$\left(\mathcal{C}_{5}^{1}\right)$

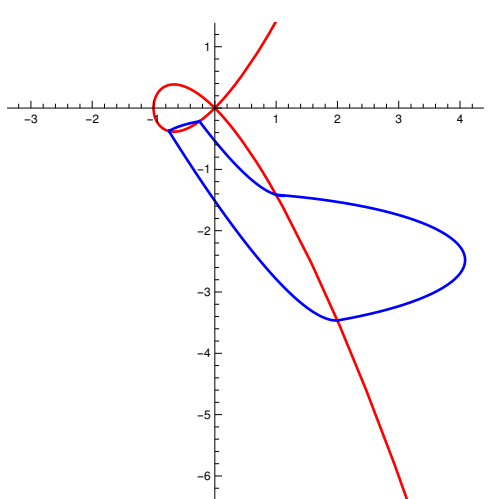

$\left(\mathcal{C}_{5}^{2}\right)$

Figure 4. The unique limit cycle of the discontinuous piecewise linear differential system $\left(\mathcal{C}_{5}^{1}\right)$ for $(5)-(7)$, and $\left(\mathcal{C}_{5}^{2}\right)$ for $(9)-(11)$ contained in three zones.

this differential system has the first integral

$$
H_{2}(x, y)=4 x^{2}+x\left(-2 y-\frac{211}{8}\right)+\frac{1}{14} y(7 y+158) .
$$

Now we consider the third linear differential center in the region $R_{3}$

$$
\dot{x}=-x-\frac{5 y}{4}+\frac{1}{2}, \quad \dot{y}=x+y-2,
$$

this differential system has the first integral

$$
H_{3}(x, y)=4(x+y)^{2}+8\left(-2 x-\frac{y}{2}\right)+y^{2} .
$$

The discontinuous piecewise linear differential system formed by the linear differential centers (1), (2) and (3) has exactly one crossing limit cycle, because the system 


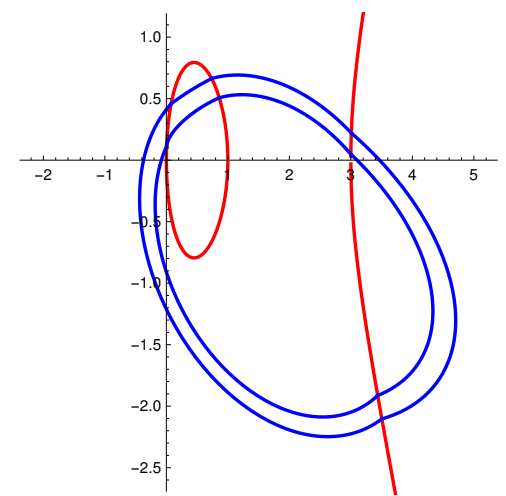

$\left(\mathcal{C}_{2}\right)$

Figure 5. The two limit cycles of the discontinuous piecewise linear differential system (12)-(14) contained in three zones.

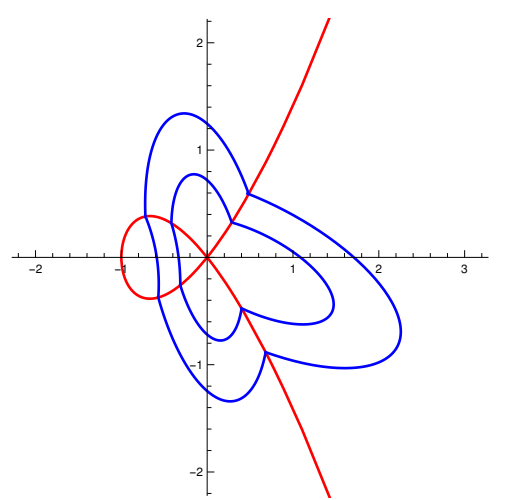

$\left(\mathcal{C}_{5}^{1}\right)$

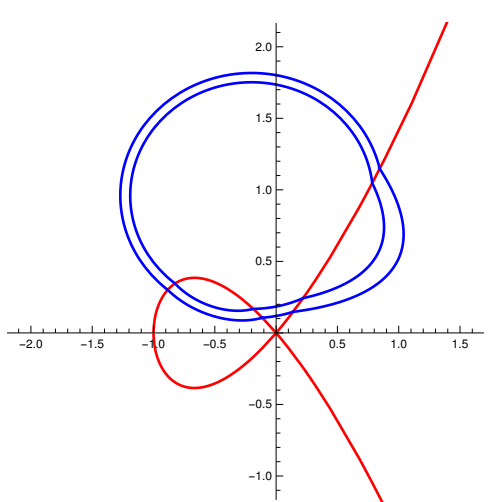

$\left(\mathcal{C}_{5}^{2}\right)$

Figure 6. The two limit cycles of the discontinuous piecewise linear differential system $\left(\mathcal{C}_{5}^{1}\right)$ for $(15)-(17)$, and $\left(\mathcal{C}_{5}^{2}\right)$ for $(18)-(20)$ contained un three zones.

of equations

$$
\begin{aligned}
& H_{1}(\alpha, \beta)-H_{1}(\gamma, \delta)=0, \\
& H_{2}(\alpha, \beta)-H_{2}(f, g)=0, \\
& H_{2}(\gamma, \delta)-H_{2}(h, k)=0, \\
& H_{3}(f, g)-H_{3}(h, k)=0, \\
& \beta^{2}-\alpha(\alpha-1)(\alpha-3)=0, \\
& \delta^{2}-\gamma(\gamma-1)(\gamma-3)=0, \\
& g^{2}-f(f-1)(f-3)=0, \\
& k^{2}-h(h-1)(h-3)=0,
\end{aligned}
$$

has a unique real solution $(\alpha, \beta, \gamma, \delta, f, g, h, k)=(5,-2 \sqrt{10}, 5,2 \sqrt{10}, 1 / 2$, $-\sqrt{5} / 2 \sqrt{2}, 1 / 2, \sqrt{5} / 2 \sqrt{2}$ ). This completes the proof of statement (a) for the class $C_{2}$. 


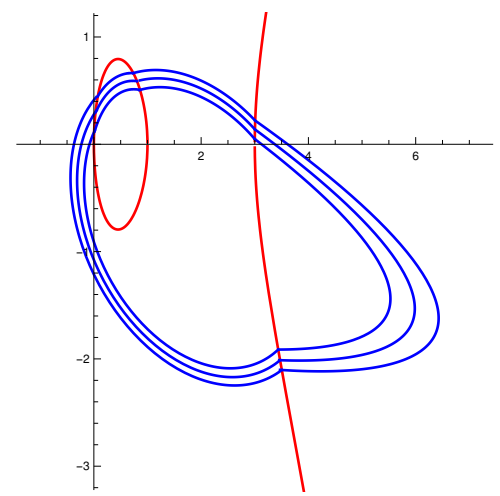

$\left(\mathcal{C}_{2}\right)$

Figure 7. The three limit cycles of the discontinuous piecewise linear differential system (21)-(23) contained in three zones.

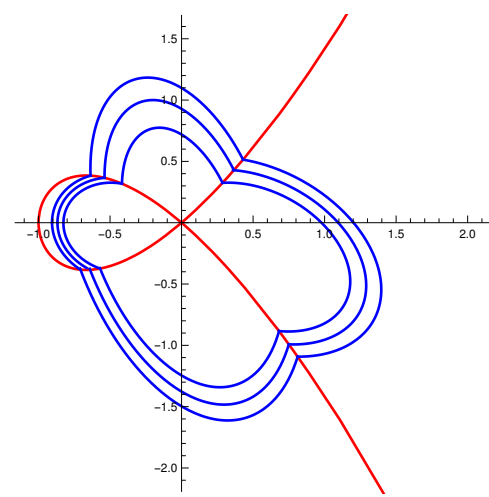

$\left(\mathcal{C}_{5}^{1}\right)$

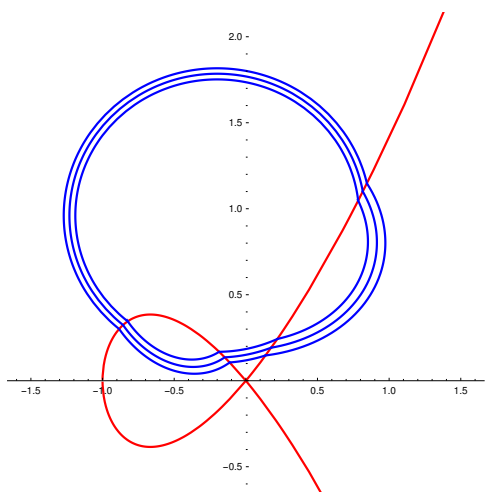

$\left(\mathcal{C}_{5}^{2}\right)$

Figure 8. The three limit cycles of the discontinuous piecewise linear differential system $\left(\mathcal{C}_{5}^{1}\right)$ for $(24)-(26)$, and $\left(\mathcal{C}_{5}^{2}\right)$ for $(27)-(29)$ contained in three zones.

Now we prove the existence of two different configurations of one crossing limit cycle for the class $C_{5}$. For the first possible configuration we consider the linear differential center

$$
\dot{x}=-\frac{x}{8}-\frac{17 y}{64}+\frac{3}{8}, \quad \dot{y}=x+\frac{y}{8}-1,
$$

in the region $R_{1}$, with its first integral

$$
H_{1}(x, y)=4\left(x+\frac{y}{8}\right)^{2}+8\left(-x-\frac{3 y}{8}\right)+y^{2} .
$$




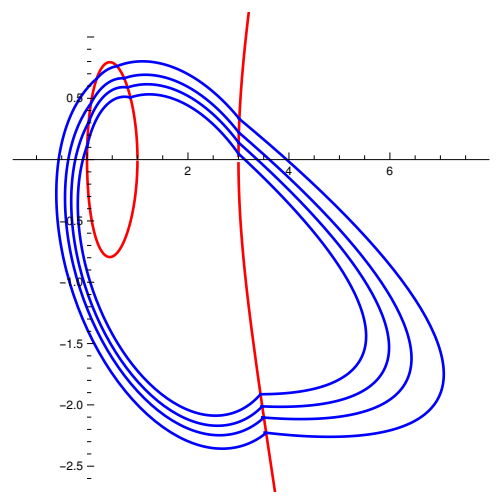

$\left(\mathcal{C}_{2}\right)$

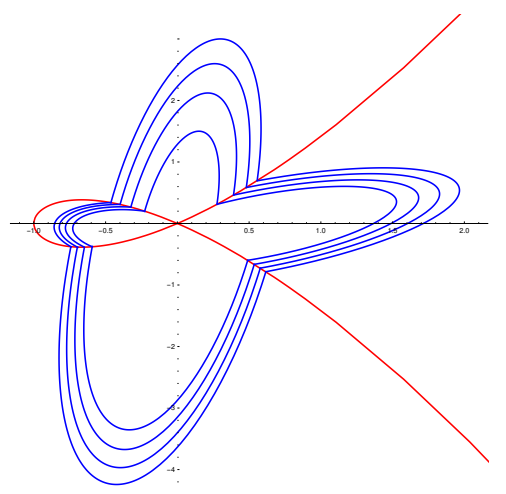

$\left(\mathcal{C}_{5}\right)$

Figure 9. The four limit cycles of the discontinuous piecewise linear differential system $\left(\mathcal{C}_{2}\right)$ for $(30)-(32)$, and $\left(\mathcal{C}_{5}\right)$ for $(33)-(35)$ contained in three zones.

In the region $R_{2}$ we consider the linear center

$$
\dot{x}=\frac{x}{4}-\frac{101 y}{16}+\frac{3800 \sqrt{2}+3584 \sqrt{5}+2203113}{320(95 \sqrt{2}+56 \sqrt{5}-4380)},
$$

$$
\dot{y}=x-\frac{y}{4}+\frac{-448604 \sqrt{2}-315662 \sqrt{5}+48 \sqrt{10}+22560621}{160(95 \sqrt{2}+56 \sqrt{5}-4380)},
$$

with its first integral

$$
\begin{aligned}
H_{2}(x, y)= & \frac{1}{40(95 \sqrt{2}+56 \sqrt{5}-4380)} \\
& \left(160(95 \sqrt{2}+56 \sqrt{5}-4380) x^{2}+x(-80(95 \sqrt{2}+56 \sqrt{5}-4380) y\right. \\
& -897208 \sqrt{2}-631324 \sqrt{5}+96 \sqrt{10}+45121242)+y(1010(95 \sqrt{2} \\
& +56 \sqrt{5}-4380) y-3800 \sqrt{2}-3584 \sqrt{5}-2203113) .
\end{aligned}
$$

In the region $R_{3}$ we consider the following linear differential center

$$
\dot{x}=-x-10 y-\frac{1}{2}, \quad \dot{y}=x+y-2
$$

this differential system has the first integral

$$
H_{3}(x, y)=4(x+y)^{2}+8\left(\frac{5}{12}\left(-3 \sqrt{2}+\frac{81}{50}-\frac{288}{25 \sqrt{5}}\right) x-y\right)+16 y^{2} .
$$

The discontinuous piecewise linear differential system formed by the linear differential centers (5), (6) and (7) has exactly one crossing limit cycle, because the system 
of equations

$$
\begin{aligned}
& H_{1}(\alpha, \beta)-H_{1}(\gamma, \delta)=0, \\
& H_{2}(\alpha, \beta)-H_{2}(f, g)=0, \\
& H_{2}(\gamma, \delta)-H_{2}(h, k)=0, \\
& H_{3}(f, g)-H_{3}(h, k)=0, \\
& \beta^{2}-\alpha^{2}(\alpha+1)=0, \\
& \delta^{2}-\gamma^{2}(\gamma+1)=0, \\
& g^{2}-f^{2}(f+1)=0, \\
& k^{2}-h^{2}(h+1)=0,
\end{aligned}
$$

has a unique real solution $(\alpha, \beta, \gamma, \delta, f, g, h, k)=(3,-6,3,6,-1 / 2,-1 /(2 \sqrt{2}),-4 / 5$, $4 /(5 \sqrt{5}))$.

For the second configuration. In the region $R_{1}$ we consider the linear differential center

$$
\dot{x}=\frac{x}{7}-\frac{785 y}{49}+\frac{70 \sqrt{50-8 \sqrt{6}}+40083}{490(\sqrt{2}-2 \sqrt{3})}, \quad \dot{y}=x-\frac{y}{7}+\frac{1}{5},
$$

which has the first integral

$$
H_{1}(x, y)=4\left(x-\frac{y}{7}\right)^{2}+8\left(\frac{x}{5}+\frac{(70 \sqrt{2}-280 \sqrt{3}-40083) y}{490(\sqrt{2}-2 \sqrt{3})}\right)+64 y^{2} .
$$

In the region $R_{2}$ we consider the linear differential center (10)

$$
\begin{aligned}
& \dot{x}=-\frac{3 x}{7}-\frac{373 y}{1764}+\frac{-23556784 \sqrt{2}-26814823 \sqrt{3}+14402472 \sqrt{6}+83393133}{80960544}, \\
& \dot{y}=x+\frac{3 y}{7}+\frac{-4861464 \sqrt{2}+3092483 \sqrt{3}+1548288 \sqrt{6}-1763763}{56448(88 \sqrt{2}-91 \sqrt{3}+15)},
\end{aligned}
$$

with its first integral

$$
H_{2}(x, y)=4\left(x+\frac{3 y}{7}\right)^{2}+\frac{y^{2}}{9}+\frac{A}{7056(88 \sqrt{2}-91 \sqrt{3}+15)(16 \sqrt{3}-3)}
$$

where

$$
\begin{aligned}
A= & 88902216 \sqrt{2}-37497657 \sqrt{3}-82428288 \sqrt{6}+153730473) x-8(7597532 \sqrt{3} \\
& +266112 \sqrt{6(259-32 \sqrt{3})}-23982207) y .
\end{aligned}
$$

In the region $R_{3}$ we consider the linear differential system

$$
\dot{x}=\frac{x}{8}-\frac{577 y}{64}+\frac{-10025 \sqrt{3}-29787}{9216}, \quad \dot{y}=x-\frac{y}{8}-\frac{1}{3},
$$

which has the first integral

$$
H_{3}(x, y)=4\left(x-\frac{y}{8}\right)^{2}+8\left(\frac{(10025 \sqrt{3}+29787) y}{9216}-\frac{x}{3}\right)+36 y^{2} .
$$

The real solutions of the system of equations (8) with the values of $H_{i}(x, y)$ with $i=1,2,3$ given for this second configuration is $(\alpha, \beta, \gamma, \delta, f, g, h, k)=(1,-\sqrt{2}, 2$, 
$-2 \sqrt{3},-1 / 4,-\sqrt{3} / 8,-3 / 4,-3 / 8)$, then the discontinuous piecewise linear differential system (9), (10) and (11) has one crossing limit cycle $\left(\mathcal{C}_{5}^{1}\right)$ of Figure 4 . This completes the proof of statement (a) for the class $C_{5}$.

Proof of statement (b) of Theorem 2. First we prove the statement for the class $C_{2}$. We consider the first linear differential center in the region $R_{1}$

$$
\dot{x}=-x-5 y-1.8035, \quad \dot{y}=x+y-0.664282,
$$

this system has the first integral

$$
H_{1}(x, y)=4(x+y)^{2}+8(1.8035 y-0.664282 x)+16 y^{2} .
$$

The second linear differential center in the region $R_{2}$ is

$$
\dot{x}=-\frac{x}{2}-\frac{10 y}{4}-1, \quad \dot{y}=x+\frac{y}{2}-\frac{3}{2},
$$

this differential system has the first integral

$$
H_{2}(x, y)=4\left(x+\frac{y}{2}\right)^{2}+8\left(y-\frac{3 x}{2}\right)+9 y^{2} .
$$

The third linear differential center in the region $R_{3}$ is

$$
\dot{x}=-2 x-8 y+0.837903, \quad \dot{y}=x-2 y-0.169396,
$$

this differential system has the first integral

$$
H_{3}(x, y)=4(x-2 y)^{2}+8(-0.169396 x-0.837903 y)+16 y^{2} .
$$

The discontinuous piecewise linear differential system formed by the linear differential centers (12), (13) and (14) have exactly two crossing limit cycles, because the system of equations (4) has two real solutions $\left(\alpha_{1}, \beta_{1}, \gamma_{1}, \delta_{1}, f_{1}, g_{1}, h_{1}, k_{1}\right)=$ (3.00039..,0.0485802.., 3.43695.., -1.91305.., 0.860569.., 0.506666..,0.00442503.., 0.114878..) and $\left(\alpha_{2}, \beta_{2}, \gamma_{2}, \delta_{2}, f_{2}, g_{2}, h_{2}, k_{2}\right)=(3.00805 . ., 0.220494 . ., 3.50419 .$. , $-2.1034 . ., 0.735716 . ., 0.663523 . ., 0.0779996 . ., 0.458408 .$.$) . This completes the proof$ of statement (b) for the class $C_{2}$.

Now we prove the existence of two different configurations of two crossing limit cycles for the class $C_{5}$. For the first configuration we consider the linear differential center

$$
\dot{x}=-\frac{6 x}{5}-\frac{369 y}{100}+0.152456, \quad \dot{y}=x+\frac{6 y}{5}-0.365572,
$$

in the region $R_{1}$, with its first integral

$$
H_{1}(x, y)=4\left(x+\frac{6 y}{5}\right)^{2}+8(-0.365572 x-0.152456 y)+9 y^{2} .
$$

In the region $R_{3}$ we consider the linear center

$$
\dot{x}=-\frac{x}{5}-\frac{29 y}{100}, \quad \dot{y}=x+\frac{y}{5},
$$

with its first integral

$$
H_{2}(x, y)=4\left(x+\frac{y}{5}\right)^{2}+y^{2} .
$$

In the region $R_{2}$ we consider the following linear differential center

$$
\dot{x}=-y-0.251587, \quad \dot{y}=x+1.93017,
$$


this differential system has the first integral

$$
H_{3}(x, y)=4 x^{2}+8(1.93017 x+0.251587 y)+4 y^{2} .
$$

The discontinuous piecewise linear differential system formed by the linear differential centers (12), (13) and (14) have exactly two crossing limit cycles, because the system of equations (8) has two real solutions $\left(\alpha_{1}, \beta_{1}, \gamma_{1}, \delta_{1}, f_{1}, g_{1}, h_{1}, k_{1}\right)=$ (0.286549..,0.325022..,0.400999.., -0.474638.., -0.416749.., 0.318275.., -0.313035.., $-0.259454 .$.$) and \left(\alpha_{2}, \beta_{2}, \gamma_{2}, \delta_{2}, f_{2}, g_{2}, h_{2}, k_{2}\right)=(0.484398 . ., 0.590171 . ., 0.681486 . .$, $-0.883698 . .,-0.719407 . ., 0.381077 . .,-0.569542 . .,-0.373673 .$.$) .$

For the second configuration. In the region $R_{1}$ we consider the linear differential center

$$
\dot{x}=-2 x-\frac{25 y}{4}+6.3854, \quad \dot{y}=x+2 y+0.134162,
$$

which has the first integral

$$
H_{1}(x, y)=4(x+2 y)^{2}+8(0.134162 x-6.3854 y)+9 y^{2} .
$$

In the region $R_{3}$ we consider the linear differential center

$$
\dot{x}=\frac{3}{2}-\frac{25 y}{16}, \quad \dot{y}=x+\frac{1}{5},
$$

with its first integral

$$
H_{2}(x, y)=4 x^{2}+8\left(\frac{x}{5}+\frac{3}{2}\right)+\frac{25 y^{2}}{4} .
$$

In the region $R_{2}$ we consider the linear differential system

$$
\dot{x}=-\frac{x}{2}-\frac{5 y}{2}+0.902952, \quad \dot{y}=x+\frac{y}{2}+0.234228,
$$

which has the first integral

$$
H_{3}(x, y)=4\left(x+\frac{y}{2}\right)^{2}+8(0.234228 x-0.902952 y)+9 y^{2} .
$$

The discontinuous piecewise linear differential system formed by the linear differential centers (18), (19) and (20) have exactly two crossing limit cycles, because the system of equations (8) has two real solutions $\left(\alpha_{1}, \beta_{1}, \gamma_{1}, \delta_{1}, f_{1}, g_{1}, h_{1}, k_{1}\right)=$ (0.844854.., 1.14753..,0.137908..,0.14711.., $-0.884123 . ., 0.300962 . .,-0.112734 .$. $0.106189 .$.$) and \left(\alpha_{2}, \beta_{2}, \gamma_{2}, \delta_{2}, f_{2}, g_{2}, h_{2}, k_{2}\right)=(0.783948 . ., 1.04708 . ., 0.219822 .$. , $0.242783 . .,-0.824415 . ., 0.345453 . .,-0.186379 . ., 0.168115 .$.$) . This completes the proof$ of statement (b) for the class $C_{2}$.

Proof of statement (c) of Theorem 2. First we prove the statement for the class $C_{2}$. We consider the first linear differential center in the region $R_{1}$

$$
\dot{x}=-3.95435 x-19.6368 y-6.39482, \quad \dot{y}=x+3.95435 y+4.13635,
$$

this system has the first integral

$$
H_{1}(x, y)=4(x+3.95435 y)^{2}+8(4.13635 x+6.39482 y)+16 y^{2} .
$$

The second linear differential center in the region $R_{2}$

$$
\dot{x}=-\frac{x}{2}-\frac{5 y}{2}-1, \quad \dot{y}=x+\frac{y}{2}-\frac{3}{2},
$$


this differential system has the first integral

$$
H_{2}(x, y)=4\left(x+\frac{y}{2}\right)^{2}+8\left(y-\frac{3 x}{2}\right)+9 y^{2} .
$$

Now we consider the third linear differential center in the region $R_{3}$

(23) $\dot{x}=-0.241343 x-1.00852 y-0.358353, \quad \dot{y}=x+0.241343 y-0.869754$,

this differential system has the first integral

$$
H_{3}(x, y)=4(x+0.241343 y)^{2}+8(0.358353 y-0.869754 x)+4 y^{2} .
$$

The discontinuous piecewise linear differential system formed by the linear differential centers (21), (22) and (23) have exactly three crossing limit cycles, because the system of equations (4) has three real solutions $\left(\alpha_{1}, \beta_{1}, \gamma_{1}, \delta_{1}, f_{1}, g_{1}, h_{1}, k_{1}\right)=$ (3.00039..,0.0485802.., 3.43695..,-1.91305..,0.860569.., 0.506666..,0.00442503.., 0.114878..), $\left(\alpha_{2}, \beta_{2}, \gamma_{2}, \delta_{2}, f_{2}, g_{2}, h_{2}, k_{2}\right)=(3.00318 . ., 0.138419 . ., 3.47181 . .,-2.01219 . .$, 0.803882.., 0.588414..,0.0319264..,0.302877..) and $\left(\alpha_{3}, \beta_{3}, \gamma_{3}, \delta_{3}, f_{3}, g_{3}, h_{3}, k_{3}\right)=$ (3.00805.., 0.220494.., 3.50419.., -2.1034.., 0.735716.., 0.663523.., 0.0779996.., 0.458408..).

Now we prove the existence of two different configurations of three crossing limit cycles for the class $C_{5}$. For the first configuration we consider the linear differential center

(24) $\dot{x}=-0.345578 x-1.11942 y-0.128163, \quad \dot{y}=x+0.345578 y-0.440337$,

in the region $R_{1}$, with its first integral

$$
H_{1}(x, y)=4(x+0.345578 y)^{2}+8(0.128163 y-0.440337 x)+4 y^{2} .
$$

In the region $R_{3}$ we consider the linear center

$$
\dot{x}=-\frac{x}{5}-\frac{29 y}{100}, \quad \dot{y}=x+\frac{y}{5},
$$

with its first integral

$$
H_{2}(x, y)=4\left(x+\frac{y}{5}\right)^{2}+y^{2} .
$$

In the region $R_{2}$ we consider the following linear differential center

(26) $\dot{x}=-0.0923038 x-1.00852 y-0.0805185, \quad \dot{y}=x+0.0923038 y+0.46371$,

this differential system has the first integral

$$
H_{3}(x, y)=4(x+0.0923038 y)^{2}+8(0.46371 x+0.0805185 y)+4 y^{2} .
$$

The discontinuous piecewise linear differential system formed by the linear differential centers (24), (25) and (26) has exactly three crossing limit cycles, because the system of equations (8) has three real solutions $\left(\alpha_{1}, \beta_{1}, \gamma_{1}, \delta_{1}, f_{1}, g_{1}, h_{1}, k_{1}\right)=$ (0.286549..,0.325022..,0.681486.., -0.883698.., -0.416749.., 0.318275.., -0.569542.., $-0.373673 ..),\left(\alpha_{2}, \beta_{2}, \gamma_{2}, \delta_{2}, f_{2}, g_{2}, h_{2}, k_{2}\right)=(0.366248 . ., 0.428095 . ., 0.749792 . .$, $-0.991822 . .,-0.538507 . ., 0.365825 . .,-0.639545 . .,-0.383969 .$.$) and \left(\alpha_{3}, \beta_{3}, \gamma_{3}, \delta_{3}, f_{3}\right.$, $\left.g_{3}, h_{3}, k_{3}\right)=(0.429988 . ., 0.514188 . ., 0.81153 . .,-1.09226 . .,-0.636378 . ., 0.383743 .$. $-0.706638 . .,-0.382736 .$.$) .$

For the second configuration. In the region $R_{1}$ we consider the linear differential center

$$
\dot{x}=-0.121241 x-2.2647 y+1.93257, \quad \dot{y}=x+0.121241 y+0.00611219,
$$


which has the first integral

$$
H_{1}(x, y)=4(x+2 y)^{2}+8(0.134162 x-6.3854 y)+9 y^{2} .
$$

In the region $R_{3}$ we consider the linear differential center

$$
\dot{x}=\frac{3}{2}-\frac{25 y}{16}, \quad \dot{y}=x+\frac{1}{5},
$$

with its first integral

$$
H_{2}(x, y)=4 x^{2}+8\left(\frac{x}{5}-\frac{3 y}{2}\right)+\frac{25 y^{2}}{4} .
$$

In the region $R_{2}$ we consider the linear differential system

(29) $\dot{x}=-0.217737 x-1.04741 y+0.515416, \quad \dot{y}=x+0.217737 y+0.350398$,

which has the first integral

$$
H_{3}(x, y)=4(x+0.217737 y)^{2}+8(0.350398 x-0.515416 y)+4 y^{2} .
$$

The discontinuous piecewise linear differential system formed by the linear differential centers (27), (28) and (29) have exactly three crossing limit cycles, because the system of equations (8) has three real solutions $\left(\alpha_{1}, \beta_{1}, \gamma_{1}, \delta_{1}, f_{1}, g_{1}, h_{1}, k_{1}\right)=$ (0.844854.., 1.14753..,0.137908.., 0.14711.., -0.884123.., 0.300962.., -0.112734.., 0.106189..), $\left(\alpha_{2}, \beta_{2}, \gamma_{2}, \delta_{2}, f_{2}, g_{2}, h_{2}, k_{2}\right)=(0.816222 . ., 1.1 . ., 0.177162 . ., 0.192216 .$. , $-0.856934 . ., 0.324128 . .,-0.147429 . ., 0.136128 .$.$) and \left(\alpha_{3}, \beta_{3}, \gamma_{3}, \delta_{3}, f_{3}, g_{3}, h_{3}, k_{3}\right)=$ (0.783948.., 1.04708.., 0.219822.., 0.242783.., -0.824415.., 0.345453..,-0.186379..,

$0.168115 .$.$) . This completes the proof of statement (c) for the class C_{5}$.

Proof of statement $(d)$ of Theorem 2. First we prove the statement for class $C_{2}$. We consider the first linear differential center in the region $R_{1}$

$$
\dot{x}=-3.95435 x-19.6368 y-6.39482, \quad \dot{y}=x+3.95435 y+4.13635,
$$

this system has the first integral

$$
H_{1}(x, y)=4(x+3.95435 y)^{2}+8(4.13635 x-6.39482 y)+16 y^{2} .
$$

The second linear differential center in the region $R_{2}$

$$
\dot{x}=-\frac{x}{2}-\frac{5 y}{2}-1, \quad \dot{y}=x+\frac{y}{2}-\frac{3}{2},
$$

this differential system has the first integral

$$
H_{2}(x, y)=4\left(x+\frac{y}{2}\right)^{2}+8\left(y-\frac{3 x}{2}\right)+9 y^{2} .
$$

Now we consider the third linear differential center in the region $R_{3}$

(32) $\dot{x}=-0.241343 x-1.00852 y-0.358353, \quad \dot{y}=x+0.241343 y-0.869754$,

this differential system has the first integral

$$
H_{3}(x, y)=4(x+0.241343 y)^{2}+8(0.358353 y-0.869754 x)+4 y^{2} .
$$

The discontinuous piecewise linear differential system formed by the linear differential centers (30), (31) and (32) have exactly four crossing limit cycles, because the system of equations (4) has four real solutions $\left(\alpha_{1}, \beta_{1}, \gamma_{1}, \delta_{1}, f_{1}, g_{1}, h_{1}, k_{1}\right)=$ (3.00039..,0.0485802.., 3.43695..,-1.91305..,0.860569..,0.506666.., 0.00442503.., 0.114878..), $\left(\alpha_{2}, \beta_{2}, \gamma_{2}, \delta_{2}, f_{2}, g_{2}, h_{2}, k_{2}\right)=(3.00318 . ., 0.138419 . ., 3.47181 . .,-2.01219 . .$, $0.803882 . ., 0.588414 . ., 0.0319264 . ., 0.302877 ..),\left(\alpha_{3}, \beta_{3}, \gamma_{3} \delta_{3}, f_{3}, g_{3}, h_{3}, k_{3}\right)=(3.00805 .$. , 
0.220494..,3.50419..,-2.1034..,0.735716..,0.663523..,0.0779996..,0.458408..) and ( $\alpha_{4}$, $\left.\beta_{4}, \gamma_{4}, \delta_{4}, f_{4}, g_{4}, h_{3} 4, k_{4}\right)=(3.01815 . ., 0.332542 . ., 3.5491 . .,-2.22883 . ., 0.593338 . .$, $0.762036 . ., 0.192725 . ., 0.66088 .$.$) .$

Now we prove the existence of two different configurations of four crossing limit cycles for the class $C_{5}$. For the first configuration we consider the linear differential center

$$
\dot{x}=0.897851 x-2.12174 y-0.62272, \quad \dot{y}=x-0.897851 y-0.620434,
$$

in the region $R_{1}$, with its first integral

$$
H_{3}(x, y)=4(x-0.897851 y)^{2}+8(0.62272 y-0.620434 x)+5.2624 y^{2} \text {. }
$$

In the region $R_{3}$ we consider the linear center

$$
\dot{x}=\frac{x}{10}-\frac{17 y}{450}, \quad \dot{y}=x-\frac{y}{10},
$$

with its first integral

$$
H_{2}(x, y)=4\left(x-\frac{y}{10}\right)^{2}+\frac{y^{2}}{9} .
$$

In the region $R_{2}$ we consider the following linear differential center

(35) $\dot{x}=-0.225709 x-1.26066 y-0.265909, \quad \dot{y}=x+0.225709 y+0.334228$,

this differential system has the first integral

$$
H_{3}(x, y)=4(x+0.225709 y)^{2}+8(0.334228 x+0.265909 y)+4.83887 y^{2} .
$$

The discontinuous piecewise linear differential system formed by the linear differential centers (33), (34) and (35) have exactly four crossing limit cycles, because the system of equations (8) has four real solutions $\left(\alpha_{1}, \beta_{1}, \gamma_{1}, \delta_{1}, f_{1}, g_{1}, h_{1}, k_{1}\right)=$ (0.275692..,0.311385..,0.490206..,-0.598415..,-0.22775..,0.200142..,-0.593285.., $-0.378363 ..),\left(\alpha_{2}, \beta_{2}, \gamma_{2} \delta_{2}, f_{2}, g_{2}, h_{2}, k_{2}\right)=(0.391233 . ., 0.461461 . ., 0.535875 . .$,

$-0.664112 . .,-0.324107 . ., 0.266457 . .,-0.647454 . .,-0.38443 ..),\left(\alpha_{3}, \beta_{3}, \gamma_{3}, \delta_{3}, f_{3}, g_{3}\right.$, $\left.h_{3}, k_{3}\right)=(0.480359 . ., 0.584453 . ., 0.577721 . .,-0.72566 . .,-0.399001 . ., 0.309322 .$. ,

$-0.696708 . .,-0.383691 .$.$) and \left(\alpha_{4}, \beta_{4}, \gamma_{4} \delta_{4}, f_{4}, g_{4}, h_{3} 4, k_{4}\right)=(0.555792 . ., 0.693247 .$. , $0.616543 . .,-0.783893 . .,-0.46287 . ., 0.339233 . .,-0.742 . .,-0.376889 .$.$) .$

\section{Proof of Theorem 3}

Proof of statement (a) of Theorem 3. First we prove the statement for the class $C_{1}$. We consider the first linear differential center in the region $R_{2}$

$$
\begin{aligned}
& \dot{x}=-\frac{x}{7}-\frac{113 y}{3136}+\frac{(\sqrt{13}+1)(-187 \sqrt{3}+192 \sqrt{13}-64)}{5376}, \\
& \dot{y}=x+\frac{y}{7}+\frac{B}{75264},
\end{aligned}
$$

with $B=(\sqrt{13}+1)(-10651 \sqrt{13}+896 \sqrt{14}-1792 \sqrt{39}-2618 \sqrt{42}+896 \sqrt{182}+18505)$. This system has the first integral 


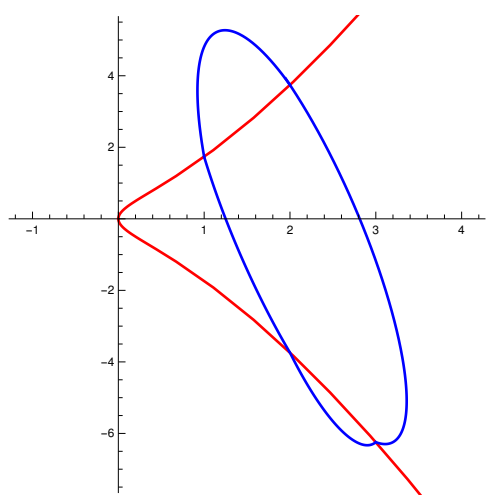

$\left(\mathcal{C}_{1}\right)$

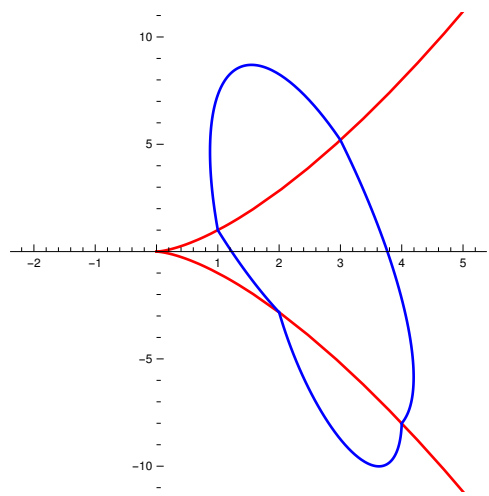

$\left(\mathcal{C}_{3}\right)$

Figure 10. The unique crossing limit cycle of the discontinuous piecewise linear differential system $\left(\mathcal{C}_{1}\right)$ for $(36)-(37)$, and $\left(\mathcal{C}_{3}\right)$ for (43)-(44) contained in two zones.

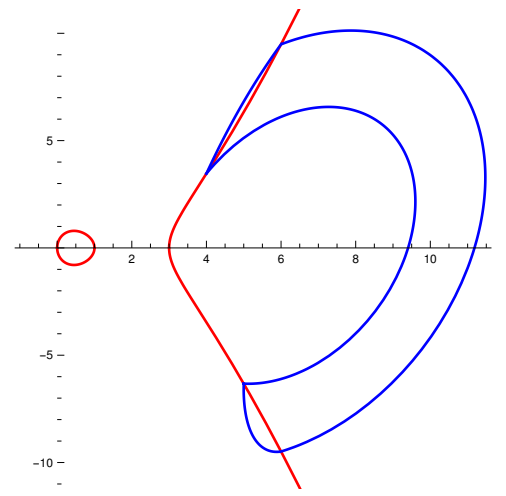

$\left(\mathcal{C}_{2}^{1}\right)$

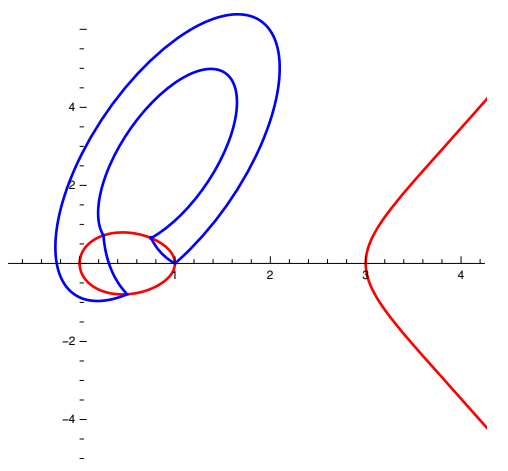

$\left(\mathcal{C}_{2}^{2}\right)$

FIGURE 11. The unique crossing limit cycle of the discontinuous piecewise linear differential system $\left(\mathcal{C}_{2}^{1}\right)$ for $(39)-(40)$, and $\left(\mathcal{C}_{2}^{2}\right)$ for (41)-(42) contained in two zones.

$$
\begin{aligned}
H_{1}(x, y)= & \frac{1}{784(\sqrt{13}-1)}\left(3136(\sqrt{13}-1) x^{2}+y(113(\sqrt{13}-1) y+14(187 \sqrt{3}\right. \\
& -192 \sqrt{13}+64))+x(896(\sqrt{13}-1) y-10651 \sqrt{13}+896 \sqrt{14} \\
& -1792 \sqrt{39}-2618 \sqrt{42}+896 \sqrt{182}+18505)) .
\end{aligned}
$$
(37)

$$
\begin{aligned}
& \dot{x}=-\frac{x}{6}-\frac{25 y}{576}-\frac{-288 \sqrt{13}+\frac{751}{\sqrt{3}}+96}{576(\sqrt{13}-1)}, \\
& \dot{y}=x+\frac{y}{6}-\frac{6009 \sqrt{13}+576 \sqrt{14}+1152 \sqrt{39}-1502 \sqrt{42}+576 \sqrt{182}-10515}{3456(\sqrt{13}-1)},
\end{aligned}
$$




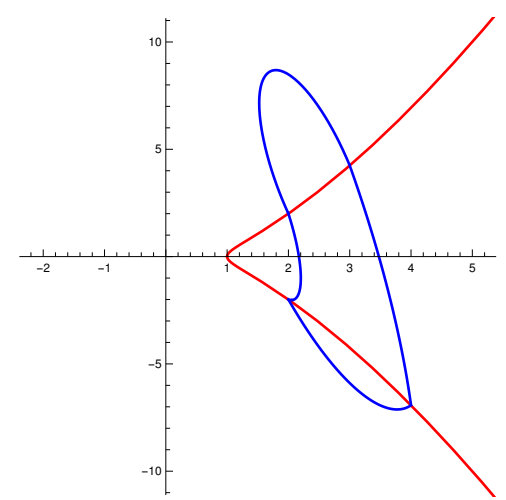

$\left(\mathcal{C}_{4}\right)$

Figure 12. The unique crossing limit cycle of the discontinuous piecewise linear differential system (45)-(46) contained in two zones.

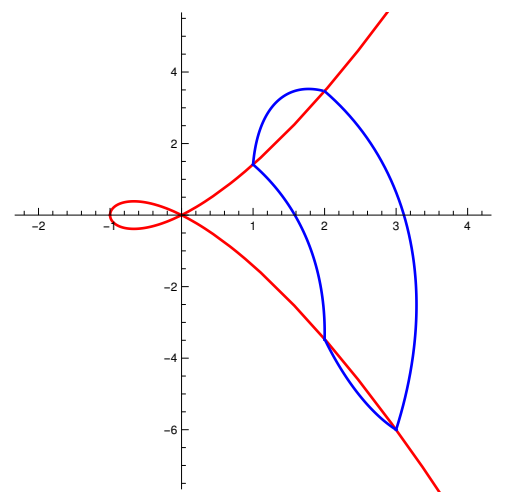

$\left(\mathcal{C}_{5}^{1}\right)$

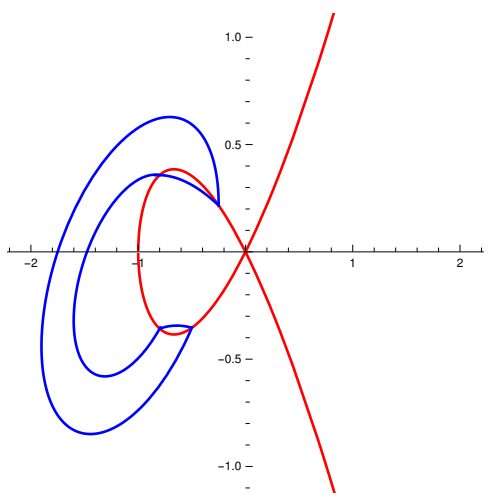

$\left(\mathcal{C}_{5}^{2}\right)$

Figure 13. The unique crossing limit cycle of the discontinuous piecewise linear differential system $\left(\mathcal{C}_{5}^{1}\right)$ for $(47)-(48)$, and $\left(\mathcal{C}_{5}^{2}\right)$ for (49)-(50) contained in two zones.

this differential system has the first integral

$$
\begin{aligned}
H_{2}(x, y)= & \frac{1}{432(\sqrt{13}-1)}\left(1728(\sqrt{13}-1) x^{2}-x(-576(\sqrt{13}-1) y+6009 \sqrt{13}\right. \\
& +576 \sqrt{14}+1152 \sqrt{39}-1502 \sqrt{42}+576 \sqrt{182}-10515)+y(75(\sqrt{13} \\
& -1) y+2(751 \sqrt{3}-864 \sqrt{13}+288))) .
\end{aligned}
$$




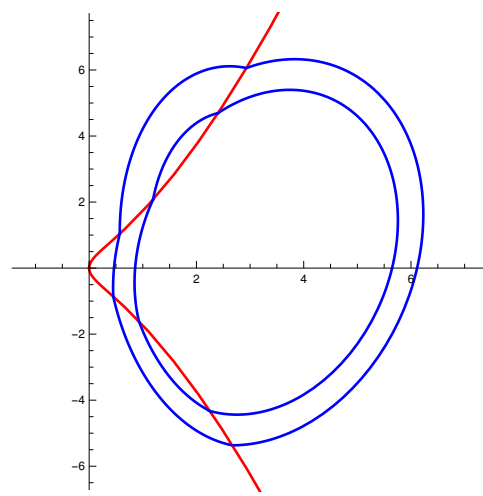

$\left(\mathcal{C}_{1}\right)$

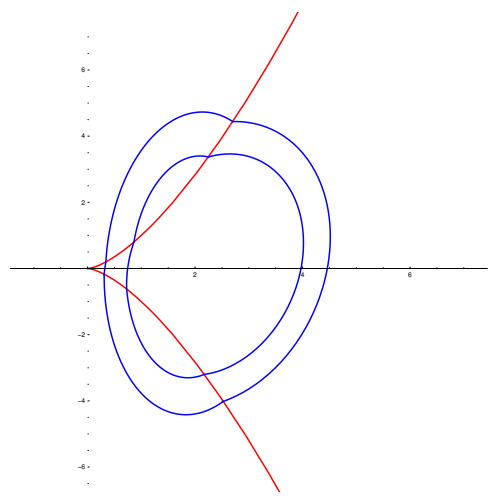

$\left(\mathcal{C}_{3}\right)$

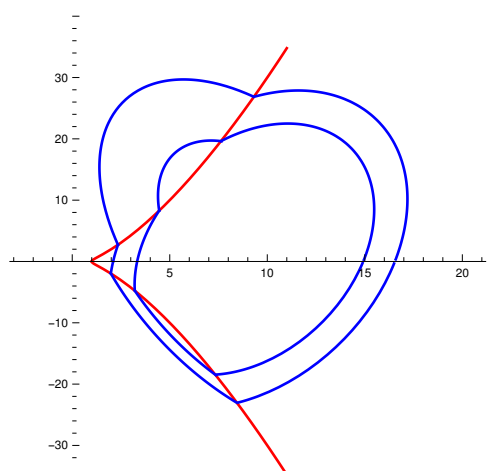

$\left(\mathcal{C}_{4}\right)$

Figure 14. The two crossing limit cycles of the discontinuous piecewise linear differential system $\left(\mathcal{C}_{1}\right)$ for $(51)-(52),\left(\mathcal{C}_{3}\right)$ for $(58)-$ $(59)$, and $\left(\mathcal{C}_{4}\right)$ for $(60)-(61)$ contained in two zones.

For the piecewise linear differential system (36)-(37) the unique real solution of the system of equations

$$
\begin{aligned}
& H_{1}\left(\alpha_{1}, \beta_{1}\right)-H_{1}\left(\gamma_{1}, \delta_{1}\right)=0, \\
& H_{1}\left(\alpha_{2}, \beta_{2}\right)-H_{1}\left(\gamma_{2}, \delta_{2}\right)=0, \\
& H_{2}\left(\alpha_{1}, \beta_{1}\right)-H_{2}\left(\alpha_{2}, \beta_{2}\right)=0, \\
& H_{2}\left(\left(\gamma_{1}, \delta_{1}\right)-H_{2}\left(\gamma_{2}, \delta_{2}\right)=0,\right. \\
& c_{i}\left(\alpha_{1}, \beta_{1}\right)=0, \quad c_{i}\left(\alpha_{2}, \beta_{2}\right)=0, \\
& c_{i}\left(\gamma_{1}, \delta_{1}\right)=0, \quad c_{i}\left(\gamma_{2}, \delta_{2}\right)=0,
\end{aligned}
$$

when $i=1$, is $\left(\alpha_{1}, \beta_{1}, \alpha_{2}, \beta_{2}, \gamma_{1}, \delta_{1}, \gamma_{2}, \delta_{2}\right)=(1, \sqrt{3}, 2,-\sqrt{14}, 2, \sqrt{14}, 3,-\sqrt{39})$.

Now we prove the statement for the class $C_{2}$. We consider the first linear differential center in the region $R_{1}$

$$
\dot{x}=\frac{x}{6}-\frac{5 y}{18}-1, \quad \dot{y}=x-\frac{y}{6}+\frac{1}{18}(12 \sqrt{3}+6 \sqrt{10}-151),
$$

this differential system has the first integral 


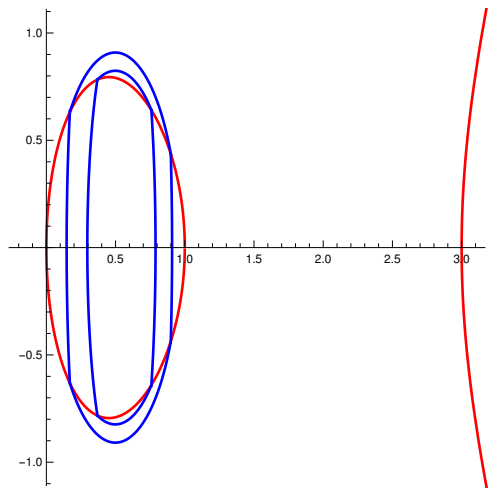

$\left(\mathcal{C}_{2}^{1}\right)$

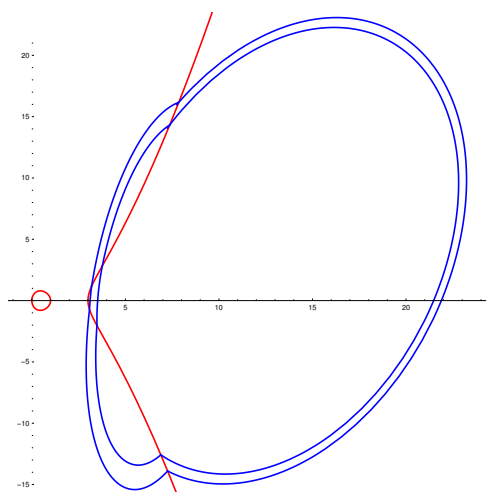

$\left(\mathcal{C}_{2}^{2}\right)$

Figure 15. The two crossing limit cycles of the discontinuous piecewise linear differential system $\left(\mathcal{C}_{2}^{1}\right)$ for $(54)-(55)$, and $\left(\mathcal{C}_{2}^{2}\right)$ for (56)-(57) contained in two zones.

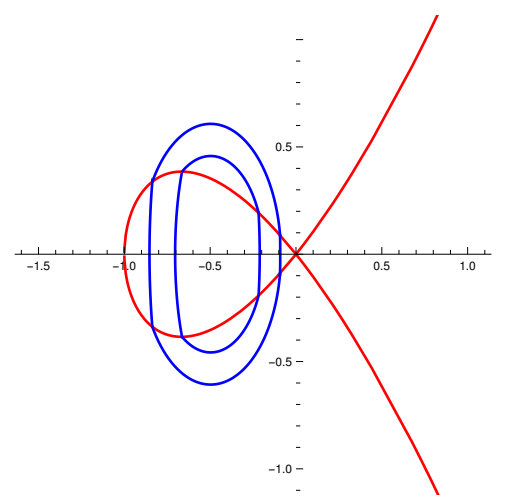

$\left(\mathcal{C}_{5}^{1}\right)$

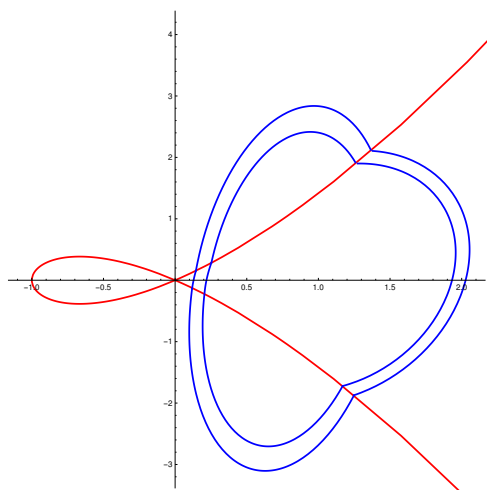

$\left(\mathcal{C}_{5}^{2}\right)$

Figure 16. The two crossing limit cycles of the discontinuous piecewise linear differential system $\left(\mathcal{C}_{5}^{1}\right)$ for $(62)-(63)$, and $\left(\mathcal{C}_{5}^{2}\right)$ for (64)-(65) contained in two zones.

$H_{1}(x, y)=\frac{2}{9}\left(18 x^{2}+2 x(-3 y+12 \sqrt{3}+6 \sqrt{10}-151)+y(5 y+36)\right)$.

The second linear differential center in the region $R_{2}$ is

$(40)$

$$
\dot{x}=\frac{x}{6}-\frac{13 y}{144}+\frac{-82 \sqrt{3}-205 \sqrt{10}-96 \sqrt{30}-5664}{4896},
$$

$$
\dot{y}=x-\frac{y}{6}+\frac{-432 \sqrt{10}+\sqrt{30(3936 \sqrt{10}+24721)}-17964}{2448},
$$

this system has the first integral 


$$
\begin{aligned}
H_{2}(x, y)= & \frac{1}{36(2 \sqrt{3}-5 \sqrt{10})}\left(144(2 \sqrt{3}-5 \sqrt{10}) x^{2}-4 x(24 \sqrt{3} y-60 \sqrt{10} y\right. \\
& +1117 \sqrt{3}-574-2649 \sqrt{10}+96 \sqrt{30}-720)+y(26 \sqrt{3} y-65 \sqrt{10} y \\
& +384 \sqrt{3}-1632 \sqrt{10})) .
\end{aligned}
$$

The unique real solution of the system of equations (38) for $i=2$, for the piecewise linear differential system (39)-(40) is $\left(\alpha_{1}, \beta_{1}, \alpha_{2}, \beta_{2}, \gamma_{1}, \delta_{1}, \gamma_{2}, \delta_{2}\right)=(4,2 \sqrt{3}, 5$, $-2 \sqrt{10}, 6,3 \sqrt{10}, 6,-3 \sqrt{10})$.

For the second configuration of the class $C_{2}$, we consider the linear system

$$
\dot{x}=\frac{x}{5}-\frac{41 y}{400}+\frac{1440 \sqrt{3}-640 \sqrt{10}-160 \sqrt{33}+2503}{3200(-3 \sqrt{3}+2 \sqrt{10}+\sqrt{33})},
$$

$$
\dot{y}=x-\frac{y}{5}+\frac{160 \sqrt{10}+\frac{2503 \sqrt{10}+960 \sqrt{30}-3200}{-3 \sqrt{3}+2 \sqrt{10}+\sqrt{33}}-4390}{6400},
$$

this differential system has the first integral

$$
\begin{aligned}
H_{1}(x, y)= & \frac{1}{800(-3 \sqrt{3}+2 \sqrt{10}+\sqrt{33})}\left(3200(-3 \sqrt{3}+2 \sqrt{10}+\sqrt{33}) x^{2}+x(13170 \sqrt{3}\right. \\
& -6277 \sqrt{10}+480 \sqrt{30}-4390 \sqrt{33}+160 \sqrt{330}-1280(-3 \sqrt{3}+2 \sqrt{10}+ \\
& \sqrt{33}) y)+2 y(164(-3 \sqrt{3}+2 \sqrt{10}+\sqrt{33}) y-1440 \sqrt{3}+640 \sqrt{10}+160 \sqrt{33} \\
& -2503)) .
\end{aligned}
$$

The second linear differential center in the region $R_{2}$ is

$$
\begin{aligned}
& \dot{x}=\frac{x}{5}-\frac{13 y}{100}+\frac{360 \sqrt{3}-160 \sqrt{10}-40 \sqrt{33}+579}{800(-3 \sqrt{3}+2 \sqrt{10}+\sqrt{33})}, \\
& \dot{y}=x-\frac{y}{5}-\frac{-3873 \sqrt{3}+4898 \sqrt{10}+1440 \sqrt{11}+480 \sqrt{30}+2449 \sqrt{33}}{3200(-3 \sqrt{3}+2 \sqrt{10}+\sqrt{33})},
\end{aligned}
$$

this system has the first integral

$$
\begin{aligned}
H_{2}(x, y)= & \frac{1}{400(-3 \sqrt{3}+2 \sqrt{10}+\sqrt{33})} 1600(-3 \sqrt{3}+2 \sqrt{10}+\sqrt{33}) x^{2}-x(640(-3 \sqrt{3} \\
& +2 \sqrt{10}+\sqrt{33}) y-3873 \sqrt{3}+4898 \sqrt{10}+1440 \sqrt{11}+480 \sqrt{30}+2449 \sqrt{33}) \\
& +4 y(52(-3 \sqrt{3}+2 \sqrt{10}+\sqrt{33}) y-360 \sqrt{3}+160 \sqrt{10}+40 \sqrt{33}-579) .
\end{aligned}
$$

The unique real solution of the system of equations (38) for $i=2$, for the piecewise linear differential system (41)-(42) is $\left(\alpha_{1}, \beta_{1}, \alpha_{2}, \beta_{2}, \gamma_{1}, \delta_{1}, \gamma_{2}, \delta_{2}\right)=(1 / 4, \sqrt{33} / 8,1 / 2$, $-\sqrt{5} /(2 \sqrt{2}), 3 / 4,3 \sqrt{3} / 8,1,0)$.

We prove the statement for the class $C_{3}$. We consider the first linear differential center in the region $R_{1}$

$$
\begin{aligned}
& \dot{x}=-\frac{x}{6}-\frac{25 y}{576}+\frac{-128 \sqrt{2}+288 \sqrt{3}+483}{-384 \sqrt{2}+576 \sqrt{3}+1344} \\
& \dot{y}=x+\frac{y}{6}+\frac{-96618 \sqrt{2}+9 \sqrt{3(58745128 \sqrt{2}+89093337)}-500192}{192384},
\end{aligned}
$$

this differential system has the first integral 


$$
\begin{aligned}
H_{1}(x, y)= & \frac{1}{144(2 \sqrt{2}-3 \sqrt{3}-7)}\left(576(2 \sqrt{2}-3 \sqrt{3}-7) x^{2}+x(192(2 \sqrt{2}-3 \sqrt{3}\right. \\
& -7) y-3770 \sqrt{2}+6861 \sqrt{3}+1152 \sqrt{6}+14875)+y(25(2 \sqrt{2}-3 \sqrt{3} \\
& -7) y-768 \sqrt{2}+1728 \sqrt{3}+2898) .
\end{aligned}
$$

The second linear differential center in the region $R_{2}$ is

$$
\begin{aligned}
& \dot{x}=-\frac{x}{9}-\frac{145 y}{5184}+\frac{134932 \sqrt{2}-69489 \sqrt{3}-77682 \sqrt{6}+250949}{577152}, \\
& \dot{y}=x+\frac{y}{9}+\frac{-23197 \sqrt{2}+45834 \sqrt{3}-1728 \sqrt{6}+79814}{5184(2 \sqrt{2}-3 \sqrt{3}-7)},
\end{aligned}
$$

this system has the first integral

$$
\begin{aligned}
H_{2}(x, y)= & \frac{1}{648(-2 \sqrt{2}-18 \sqrt{3}+6 \sqrt{6}-20)}\left(5184(-\sqrt{2}-9 \sqrt{3}+3 \sqrt{6}-10) x^{2}\right. \\
& +x(1152(-\sqrt{2}-9 \sqrt{3}+3 \sqrt{6}-10) y+7645 \sqrt{2}+193608 \sqrt{3}+332692 \\
& -67863 \sqrt{6})+y(145(-\sqrt{2}-9 \sqrt{3}+3 \sqrt{6}-10) y+2304 \sqrt{2}+10755 \sqrt{3} \\
& -6912 \sqrt{6}+41343)) .
\end{aligned}
$$

This piecewise linear differential centers has a unique real solution of the system of equations (38) for $i=3$, which is $\left(\alpha_{1}, \beta_{1}, \alpha_{2}, \beta_{2}, \gamma_{1}, \delta_{1}, \gamma_{2}, \delta_{2}\right)=(1,1,2,-2 \sqrt{2}, 3,3 \sqrt{3}$, $4,-8)$.

Now we prove the statement for the class $C_{4}$. We consider the linear differential center in the region $R_{1}$

$$
\dot{x}=-\frac{x}{9}-\frac{181 y}{8100}+\frac{2}{9}, \quad \dot{y}=x+\frac{y}{9}+\frac{\sqrt{2}}{3}-\frac{2071}{540}+\frac{8}{3 \sqrt{3}},
$$

this differential system has the first integral

$$
H_{1}(x, y)=4 x^{2}+\frac{2}{135} x(60 y+180 \sqrt{2}+480 \sqrt{3}-2071)+\frac{y(181 y-3600)}{2025} .
$$

The second linear differential center in the region $R_{2}$ is

$$
\begin{aligned}
& \dot{x}=-\frac{x}{8}-\frac{y}{32}+\frac{66 \sqrt{2}-62 \sqrt{3}-18 \sqrt{6}+525}{1128}, \\
& \dot{y}=x+\frac{y}{8}+\frac{-600 \sqrt{6}+8 \sqrt{6(232 \sqrt{6}+2555)}-12627}{4512},
\end{aligned}
$$

this system has the first integral

$$
\begin{aligned}
H_{2}(x, y)= & \frac{1}{8(-15 \sqrt{2}-4 \sqrt{3}+6 \sqrt{6}+24)} 32(-15 \sqrt{2}-4 \sqrt{3}+6 \sqrt{6}+24) x^{2}+ \\
& 2 x(4(-15 \sqrt{2}-4 \sqrt{3}+6 \sqrt{6}+24) y+1521 \sqrt{2}+556 \sqrt{3}-666 \sqrt{6} \\
& -2456)+y((-15 \sqrt{2}-4 \sqrt{3}+6 \sqrt{6}+24) y-8(-51 \sqrt{2}-16 \sqrt{3} \\
& +24 \sqrt{6}+76)) .
\end{aligned}
$$

In this case the unique real solution of the system of equations (38) for $i=4$, is $\left(\alpha_{1}, \beta_{1}, \alpha_{2}, \beta_{2}, \gamma_{1}, \delta_{1}, \gamma_{2}, \delta_{2}\right)=(2,2,2,-2,3,3 \sqrt{2}, 4,-4 \sqrt{3})$. 
We prove the statement for the first configuration of the class $C_{5}$. We consider the linear differential center in the region $R_{3}$

$$
\begin{aligned}
& \dot{x}=-\frac{x}{6}-\frac{13 y}{144}+\frac{53 \sqrt{2}+1134}{4896}, \\
& \dot{y}=x+\frac{y}{6}+\frac{-114 \sqrt{2}+91 \sqrt{3}-24 \sqrt{6}+843}{72(\sqrt{2}-6)},
\end{aligned}
$$

this system has the first integral

$$
\begin{aligned}
H_{1}(x, y)= & \frac{1}{36(\sqrt{2}-6)}\left(144(\sqrt{2}-6) x^{2}-4 x(-12(\sqrt{2}-6) y+114 \sqrt{2}-91 \sqrt{3}\right. \\
& +24 \sqrt{6}-843)+y(13(\sqrt{2}-6) y-48 \sqrt{2}+394)) .
\end{aligned}
$$

The second linear differential center in the region $R_{1}$ is

$$
\begin{aligned}
& \dot{x}=\frac{x}{6}-\frac{5 y}{18}+\frac{1}{612}(-89 \sqrt{2}-636), \\
& \dot{y}=x-\frac{y}{6}+\frac{1}{306}(432 \sqrt{3}+178 \sqrt{3(\sqrt{3}+2)}-795),
\end{aligned}
$$

this differential system has the first integral

$$
\begin{aligned}
H_{2}(x, y)= & \frac{1}{9(\sqrt{2}-6)}\left(36(\sqrt{2}-6) x^{2}-4 x(3(\sqrt{2}-6) y+141 \sqrt{2}+142 \sqrt{3}\right. \\
& +6 \sqrt{6}-312)+2 y(5(\sqrt{2}-6) y+6 \sqrt{2}-214)) .
\end{aligned}
$$

For this piecewise linear differential centers the unique real solution of system (38) when $i=5$ is $\left(\alpha_{1}, \beta_{1}, \alpha_{2}, \beta_{2}, \gamma_{1}, \delta_{1}, \gamma_{2}, \delta_{2}\right)=(1, \sqrt{2}, 2,-2 \sqrt{3}, 2,2 \sqrt{3}, 3,-6)$. Hence, the discontinuous piecewise linear differential system (47)-(48) has a unique crossing limit cycle, see $\left(\mathcal{C}_{5}^{1}\right)$ of Figure 13.

Finally we prove the statement for the second configuration of the class $C_{5}$.In the region $R_{3}$ we consider the linear differential center

$$
\dot{x}=\frac{x}{2}-\frac{5 y}{4}+\frac{2}{5}, \quad \dot{y}=x-\frac{y}{2}+\frac{1}{640}(96 \sqrt{2}+88 \sqrt{3}+365),
$$

this system has the first integral

$$
H_{1}(x, y)=4 x^{2}+\frac{1}{80} x(-320 y+96 \sqrt{2}+88 \sqrt{3}+365)+\frac{1}{5} y(25 y-16) .
$$

In the region $R_{2}$ we consider the linear center

$$
\dot{x}=-\frac{x}{4}-\frac{y}{8}+\frac{-22000 \sqrt{2}-3000 \sqrt{3}+34816 \sqrt{5}+24585}{640(275 \sqrt{2}+75 \sqrt{3}-272 \sqrt{5}},
$$

$$
\dot{y}=x+\frac{y}{4}+\frac{2400 \sqrt{2}-2200 \sqrt{3}-\frac{4125(149 \sqrt{11-4 \sqrt{6}}+200)}{275 \sqrt{2}+75 \sqrt{3}-272 \sqrt{5}}+62593}{108800},
$$


this system has the first integral

$$
\begin{aligned}
H_{2}(x, y)= & \frac{1}{800(275 \sqrt{2}+75 \sqrt{3}-272 \sqrt{5})}\left(3200(275 \sqrt{2}+75 \sqrt{3}-272 \sqrt{5}) x^{2}\right. \\
& +x(1600(275 \sqrt{2}+75 \sqrt{3}-272 \sqrt{5}) y+940225 \sqrt{2}+312300 \sqrt{3} \\
& -1001488 \sqrt{5}-25000 \sqrt{6}-38400 \sqrt{10}+35200 \sqrt{15})+10 y(40(275 \sqrt{2} \\
& +75 \sqrt{3}-272 \sqrt{5}) y+22000 \sqrt{2}+3000 \sqrt{3}-34816 \sqrt{5}-24585)) .
\end{aligned}
$$

For this piecewise linear differential centers the unique real solution of system (38) when $i=5$ is $\left(\alpha_{1}, \beta_{1}, \alpha_{2}, \beta_{2}, \gamma_{1}, \delta_{1}, \gamma_{2}, \delta_{2}\right)=(-1 / 4, \sqrt{3} / 8,-4 / 5,4 /(5 \sqrt{5}),-1 / 2$, $-1 /(2 \sqrt{2}),-4 / 5,-4 /(5 \sqrt{5})$. Then the discontinuous piecewise linear differential system (49)-(50) has exactly one crossing limit cycle, see $\left(\mathcal{C}_{5}^{2}\right)$ of Figure 13 . This completes the proof of statement (a) of Theorem 3.

Proof of statement (b) of Theorem 3. First we prove the statement for class $C_{1}$. In the region $R_{1}$ we consider the linear differential center

(51)

$$
\dot{x}=-0.0327708 x-0.167012 y+0.202324, \quad \dot{y}=x+0.0327708 y-2.82026,
$$

its first integral is

$$
H_{1}(x, y)=4(x+0.0327708 y)^{2}+8(-2.82026 x-0.202324 y)+0.663752 y^{2} .
$$

The second linear differential center in the region $R_{2}$ is

$$
\dot{x}=\frac{x}{10}-\frac{13 y}{50}-\frac{1}{5}, \quad \dot{y}=x-\frac{y}{10}-\frac{16}{5},
$$

its first integral is

$$
H_{2}(x, y)=4\left(x-\frac{y}{10}\right)^{2}+8\left(\frac{y}{5}-\frac{16 x}{5}\right)+y^{2} .
$$

For the piecewise linear differential system (51)-(52) the real solutions of the system of equations

$$
\begin{aligned}
& H_{1}\left(\alpha_{i}, \beta_{i}\right)-H_{1}\left(\gamma_{i}, \delta_{i}\right)=0, \\
& H_{1}\left(f_{i}, g_{i}\right)-H_{1}\left(h_{i}, k_{i}\right)=0, \\
& H_{2}\left(\alpha_{i}, \beta_{i}\right)-H_{1}\left(f_{i}, g_{i}\right)=0, \\
& H_{2}\left(\gamma_{i}, \delta_{i}\right)-H_{1}\left(h_{i}, k_{i}\right)=0, \\
& c_{s}\left(\alpha_{i}, \beta_{i}\right)=c_{s}\left(\gamma_{i}, \delta_{i}\right)=0, \quad i=1,2, \\
& c_{s}\left(f_{i}, g_{i}\right)=c_{s}\left(h_{i}, k_{i}\right)=0 .
\end{aligned}
$$

when $s=1$, is $\left(\alpha_{1}, \beta_{1}, \gamma_{1}, \delta_{1}, f_{1}, g_{1}, h_{1}, k_{1}\right)=(0.571172 . ., 1.04103 . ., 2.93153 . ., 6.0596 .$. , 0.449711.., -0.861917.., 2.66907.., -5.36724..) and $\left(\alpha_{2}, \beta_{2}, \gamma_{2}, \delta_{2}, f_{2}, g_{2}, h_{2}, k_{2}\right)=$ (1.19066.., 2.07275.., 2.40283.., 4.69567.., 0.928007..,-1.60885.., 2.25189..,-4.32923..). Hence these linear differential centers have exactly two crossing limit cycles, see $\left(\mathcal{C}_{1}\right)$ of Figure 14.

Now we prove the statement for the class $C_{2}$. In the region $R_{1}$ we consider the linear differential center

$$
\dot{x}=-0.252669 y, \quad \dot{y}=x-0.498272,
$$

its first integral is

$$
H_{1}(x, y)=4 x^{2}-3.98617 x+1.01067 y^{2}
$$


The second linear differential center in the region $R_{2}$ is

$$
\dot{x}=-\frac{y}{25}, \quad \dot{y}=x-\frac{1}{2},
$$

its first integral is

$$
H_{2}(x, y)=4 x^{2}-4 x+\frac{4 y^{2}}{25} .
$$

For these centers the real solutions of system (53) when $s=2$ are $(0.898773 . ., 0.43723 .$. , $0.169784 . ., 0.631617 . ., 0.898773 . .,-0.43723 . ., 0.169784 . .,-0.631617 .$.$) and (0.758688 . .$, 0.640578..,0.368993..,0.782685..,0.758688..,-0.640578..,0.368993..,-0.782685..). Hence the discontinuous piecewise linear differential system (54)-(55) has two crossing limit cycles, see $\left(\mathcal{C}_{2}^{1}\right)$ of Figure 15 .

For the second configuration of the class $C_{2}$ we consider the linear center (56) $\dot{x}=0.057143 x-0.0550264 y-0.0521176, \quad \dot{y}=x-0.057143 y-0.805757$, in the region $R_{1}$, its first integral is

$$
H_{1}(x, y)=4(x-0.057143 y)^{2}+8(0.0521176 y-0.805757 x)+0.207044 y^{2} .
$$

The second linear differential center in the region $R_{2}$ is

$$
\dot{x}=\frac{x}{10}-\frac{17 y}{80}-\frac{1}{10}, \quad \dot{y}=x-\frac{y}{10}-\frac{11}{10},
$$

its first integral is

$$
H_{2}(x, y)=4\left(x-\frac{y}{10}\right)^{2}+8\left(\frac{y}{10}-\frac{11 x}{10}\right)+\frac{81 y^{2}}{100} \text {. }
$$

The real solutions of system (53) for these centers are $(0.253068 . ., 0.283285 . ., 1.26272 .$. , $1.89942 . ., 0.204637 . .,-0.224601 . ., 1.16927 . .,-1.72215 .$.$) and (0.145203 . ., 0.155388 . .$, 1.36881.., 2.10673..,0.119104.., -0.125997.., 1.24855..,-1.87223..). Hence the discontinuous piecewise linear differential system (56)-(57) has two crossing limit cycles, see $\left(\mathcal{C}_{2}^{2}\right)$ of Figure 15 .

For the class $C_{3}$ and in the region $R_{1}$ we consider the center

(58) $\dot{x}=0.0333015 x-0.132045 y-0.0410184, \quad \dot{y}=x-0.0333015 y-1.97694$, its first integral is

$$
H_{1}(x, y)=4(x-0.0333015 y)^{2}+8(0.0410184 y-1.97694 x)+0.523744 y^{2} .
$$

The second linear differential center in the region $R_{2}$ is

$$
\dot{x}=0.1 x-0.26 y-0.2, \quad \dot{y}=x-0.1 y-2.3,
$$

its first integral is

$$
H_{2}(x, y)=4(x-0.1 y)^{2}+8(0.2 y-2.3 x)+y^{2} .
$$

The real solutions of system (53) when $s=3$ are (0.340737.., 0.198898.., 2.70162.., 4.44056..,0.308678.., -0.171498.., 2.52616.., -4.01504..) and (0.862714.., 0.801309.., 2.24826.., 3.37109.., 0.727481.., - 0.620487.., 2.17185.., - 3.2007..). Hence the discontinuous piecewise linear differential system (58)-(59) has two crossing limit cycles, see $\left(\mathcal{C}_{3}\right)$ of Figure 14 .

We prove the statement for the class $C_{4}$. In the region $R_{1}$ we consider the center (60) $\dot{x}=-0.142331 x-0.0908868 y+1.59716, \quad \dot{y}=x+0.142331 y-9.92891$, 
its first integral is

$$
H_{1}(x, y)=4(x+0.142331 y)^{2}+8(-9.92891 x-1.59716 y)+0.282515 y^{2} .
$$

We consider the second linear differential center in the region $R_{2}$

$$
\dot{x}=\frac{x}{10}-\frac{y}{10}-\frac{7}{10}, \quad \dot{y}=x-\frac{y}{10}-\frac{44}{5},
$$

its first integral is

$$
H_{2}(x, y)=4\left(x-\frac{y}{10}\right)^{2}+8\left(\frac{7 y}{10}-\frac{44 x}{5}\right)+\frac{9 y^{2}}{25} .
$$

The two real solutions of system (53) when $s=4$ are $(2.34486 . ., 2.71929 . ., 9.31289 .$. , 26.851.., 1.97922.., -1.95854.., 8.44735..,-23.0527..) and (4.45798.., 8.2899.., 7.62439.., 19.6236.., 3.20672.., $-4.7636 . ., 7.33329 . .,-18.455 .$.$) . Hence the discontinuous piece-$ wise linear differential system $(60)-(61)$ has two crossing limit cycles, see $\left(\mathcal{C}_{4}\right)$ of Figure 14.

For the first configuration of the class $C_{5}$. We consider the linear differential center

$$
\dot{x}=-0.453205 y, \quad \dot{y}=x+0.497252,
$$

in the region $R_{3}$ with its first integral

$$
H_{1}(x, y)=4 x^{2}+3.97802 x+1.81282 y^{2} .
$$

We consider the second linear differential center in the region $R_{2}$

$$
\dot{x}=-\frac{y}{10}, \quad \dot{y}=x+\frac{1}{2},
$$

its first integral is

$$
H_{2}(x, y)=4 x^{2}+4 x+\frac{2 y^{2}}{5} .
$$

The two real solutions of system (53) when $s=5$ are $(-0.0927079 . ., 0.088306 .$. , $-0.837019 . ., 0.337912 . .,-0.0927079 . .,-0.088306 . .,-0.837019 . .,-0.337912 .$.$) and$ $(-0.217826 . ., 0.192647 . .,-0.663868 . ., 0.38489 . .,-0.217826 . .,-0.192647 . .,-0.663868 .$. $-0.38489 .$.$) . Hence, the discontinuous piecewise linear differential system (62)-(63)$ has two crossing limit cycles, see $\left(\mathcal{C}_{5}\right)$ of Figure 16.

Finally we prove the statement for the second configuration of the class $C_{5}$. In the region $R_{3}$ we consider the linear differential center

(64) $\dot{x}=0.057143 x-0.0550264 y-0.0521176, \quad \dot{y}=x-0.057143 y-0.805757$, its first integral is

$$
H_{1}(x, y)=4(x-0.057143 y)^{2}+8(0.0521176 y-0.805757 x)+0.207044 y^{2} .
$$

We consider the second linear differential center in the region $R_{1}$

$$
\dot{x}=\frac{x}{10}-\frac{17 y}{80}-\frac{1}{10}, \quad \dot{y}=x-\frac{y}{10}-\frac{11}{10},
$$

its first integral is

$$
H_{2}(x, y)=4\left(x-\frac{y}{10}\right)^{2}+8\left(\frac{y}{10}-\frac{11 x}{10}\right)+\frac{81 y^{2}}{100} \text {. }
$$


The two real solutions of system (53) when $s=5$ are $(0.253068 . ., 0.283285 . ., 1.26272 .$. , $1.89942 . ., 0.204637 . .,-0.224601 . ., 1.16927 . .,-1.72215 .$.$) and (0.145203 . ., 0.155388 . .$, 1.36881.., 2.10673..,0.119104.., -0.125997.., 1.24855..,-1.87223..). Hence the discontinuous piecewise linear differential system (64)-(65) has two crossing limit cycles, see $\left(\mathcal{C}_{5}^{2}\right)$ of Figure 16.

\section{REFERENCES}

[1] J.C. Artes, J. Llibre, J.C. Medrado and M.A. Teixeira, Piecewise linear differential systems with two real saddles, Math. Comput. Simulation 95 (2014), 13-22.

[2] S. BanerJee And G. Verghese, Nonlinear phenomena in power electronics. Attractors, bifurcations chaos and nonlinear control, Wiley-IEEE Press, New York, 2001.

[3] R.Benterki And J. LLiBre, The limit cycles of discontinuous piecewise linear differential systems formed by centers and separated by irreducible cubic curves I, preprint, 2019.

[4] R. Bix, Conics and cubics, Undergraduat Texts in Mathematics, Second Edition, Springer, 2006.

[5] R.D. EuzÉBIO AND J. LliBRE, On the number of limit cycles in discontinuous piecewise linear differential systems with two pieces separated by a straight line, J. Math. Anal. Appl. 424 (2015), 475-486.

[6] E. Freire, E. Ponce, F. Rodrigo And F. TORres, Bifurcation sets of continuous piecewise linear systems with two zones, Int. J. Bifurcation and Chaos. 8 (1998), 2073-2097.

[7] E. Freire, E. Ponce ANd F. TORRes, Canonical discontinuous planar piecewise linear systems, SIAM J. Appl. Dyn. Syst. 11 (2012), 181-211.

[8] M. Han AND W. Zhang, On Hopf bifurcation in non-smooth planar systems, J. Differential Equations. 248 (2010), 2399-2416.

[9] S.M. HUAN AND X.S. YANG, On the number of limit cycles in general planar piecewise linear systems, Disc. Cont. Dyn. Syst. 32 (2012), 2147-2164.

[10] J. J. Jimenez Ruiz, J. Llibre and J. C. Medrado., Crossing limit cycles for a class of piecewise linear differential centers separated by a conic., Preprint, 2019.

[11] J. J. Jimenez Ruiz, J. Llibre and J. C. Medrado., Crossing limit cycles for piecewise linear differential centers separated by a reducible cubic curve, Preprint, 2019.

[12] R. I. LEINE AND H. NiJMEIJER, Dynamics and bifurcations of non-smooth mechanical systems, Lecture Notes in Applied and Computational Mechanics, 18, Springer-Verlag, Berlin, 2004.

[13] D. LiBerzon, Switching in systems and control: Foundations and Applications, Birkhäuser, Boston, 2003.

[14] J. Llibre, D. D. Novaes And M. A. Teixeira, Maximum number of limit cycles for certain piecewise linear dynamical systems, Nonlinear Dynam, 82 (2015), 1159-1175.

[15] J. Llibre And E. Ponce, Three nested limit cycles in discontinuous piecewise linear differential systems with two zones, Dyn. Contin. Discr. Impul. Syst., Ser. B 19 (2012), 325-335.

[16] J. Llibre And M. A. Teixeira, Piecewise linear differential systems with only centers can create limit cycles?, Nonlinear Dynam, 91 (2018), 249-255.

[17] R. Lum And L.O. ChuA, Global propierties of continuous piecewise-linear vector fields. Part I: Simplest case in $\mathbb{R}^{2}$, Int. J. of Circuit Theory and Appl. 19 (1991), 251-307.

[18] R. Lum And L.O. ChuA, Global propierties of continuous piecewise linear vector fields. Part II: Simplest case in $\mathbb{R}^{2}$, Int. J. of Circuit Theory and Appl. 19 (1992), 9-46.

[19] S. Shui, X. Zhang And J. Li, The qualitative analysis of a class of plana Filippov systems, Nonlinear Anal. 73 (2010), 1277-1288.

1 and ${ }^{2}$ Département de Mathématiques, Université Mohamed El Bachir El Ibrahimi, Bordj Bou Arréridj 34265, El Anasser, Algeria

Email address: r.benterki@univ-bba.dz and damine.loubna@univ-bba.dz

3 Departament de Matematiques, Universitat Autònoma de Barcelona, 08193 Bellaterra, Barcelona, Catalonia, Spain

Email address: jllibre@mat.uab.cat 